\title{
Rogue Waves of Nonlinear Schrödinger Equation with Time-Dependent Linear Potential Function
}

\author{
Ni Song and Yakui Xue \\ Department of Mathematics, North University of China, Taiyuan, Shanxi 030051, China \\ Correspondence should be addressed to Yakui Xue; xyk5152@163.com
}

Received 23 November 2015; Accepted 24 January 2016

Academic Editor: Amit Chakraborty

Copyright (C) 2016 N. Song and Y. Xue. This is an open access article distributed under the Creative Commons Attribution License, which permits unrestricted use, distribution, and reproduction in any medium, provided the original work is properly cited.

\begin{abstract}
The rogue waves of the nonlinear Schrödinger equation with time-dependent linear potential function are investigated by using the similarity transformation in this paper. The first-order and second-order rogue waves solutions are obtained and the nonlinear dynamic behaviors of these solutions are discussed in detail. In addition, the amplitudes of the rogue waves under the effect of the gravity field and external magnetic field changing with the time are analyzed by using numerical simulation. The results can be used to study the matter rogue waves in the Bose-Einstein condensates and other fields of nonlinear science.
\end{abstract}

\section{Introduction}

The rogue wave is giant single wave which was firstly found in the ocean [1]. The amplitude of this wave is two to three times higher than those of its surrounding waves. The key feature of the rogue wave is that it "appears from nowhere" and "disappears without a trace" [2]. The most terrible thing is that it is very dangerous for sailors because it can appear unexpectedly and form larger amplitude in one minute to shred a boat. Beyond oceanic expanses, the rogue wave has been also found in optical fibers [3], Bose-Einstein condensates (BECs) [4], superfluids [5], and so on. However, it is very difficult to explain the rogue waves by using the linear theories based on the superposition principles. The nonlinear theories of ocean waves [6-8] can be used to explain why the rogue waves can appear from nowhere. In recent years, it becomes an important issue for ones to study the rogue waves theoretically in the fields of the nonlinear science [913]. The Darboux transformation (DT) [14, 15], the similarity transformation, and the numerical simulation $[13,16-18]$ were used to analyze the occurrence of the rogue waves and the larger amplitudes. One of the important known models for the rogue waves is the nonlinear Schrödinger (NLS) equation which is a foundational model in describing numerous nonlinear physical phenomena and the first-order rational solution was derived by Peregrine [19] and the second-order one was obtained by using the modified Darboux transformation. Cheng et al. [20] show the controllable rogue waves in coupled NLS with varying potential functions and nonlinearities. Wu et al. [21] derive the evolution of optical solitary waves in a generalized NLS equation with variable coefficients.

Since three affirmative observations on Bose-Einstein condensates in ultracold rubidium [22], lithium [23], and sodium [24] atomic gases were reported, respectively, in 1995, researches on the behaviors of the macroscopical quantum and dynamic evolution of BECs are more active and competitive than before in both experiment and theory aspects. Ivancevic and Reid [25] show that the NLS equation is fundamental for quantum turbulence, while its closed form solutions include shock-waves, solitons, and rogue waves. Zhu [26] gives nonlinear tunneling for controllable rogue waves in two-dimensional graded-index waveguides.

A new area of physics, namely, nonlinear matter waves and nonlinear atomic optics, was originated. Generation and dynamics of solitary wave pulses in BECs are one of the most important related problems. In the approximation of the mean field, the evolution characteristics of the nonlinear excitations for BECs can be described by the Gross-Pitaevskii equation $[27,28]$ which is also called the nonlinear Schrödinger equation. Particularly, in the gravity field, the evolution characteristics of the nonlinear excitations 
for BECs, which change with the time under the external magnetic field, can be described by the continuous GrossPitaevskii equation [29]:

$$
\begin{aligned}
i \hbar \frac{\partial \Psi}{\partial T}= & -\frac{\hbar^{2}}{2 m} \frac{\partial^{2} \Psi}{\partial X^{2}}+X\left[m g+B \cos \left(\omega_{1} T\right)\right] \Psi \\
& +\frac{4 \pi \hbar^{2} a}{m}|\Psi|^{2} \Psi
\end{aligned}
$$

where $\Psi(X, T)$ is the wave function of Bose-Einstein condensates, $m$ is atomic mass, $a$ is the scattering length between atoms, $m g$ is the gravity field, $B \cos \left(\omega_{1} T\right)$ is the timedependent external magnetic field, $B$ is the time-dependent field parameter, and $\omega_{1}$ is the frequency of external magnetic field.

To simplify (1), we introduce the following dimensionless transformation:

$$
\begin{gathered}
t=\frac{\hbar}{m l_{0}^{2}} T, \\
x=\frac{1}{L_{0}} X, \\
\varphi=\frac{1}{\sqrt{n_{0}}} \Psi .
\end{gathered}
$$

Then, (1) can be rewritten as

$$
i \frac{\partial \varphi}{\partial t}+\frac{1}{2} \frac{\partial^{2} \varphi}{\partial x^{2}}+x[b+l \cos (\omega t)] \varphi+\mu|\varphi|^{2} \varphi=0,
$$

where

$$
\begin{gathered}
b=-\frac{m^{2} l_{0}^{3} g}{\hbar^{2}}, \\
l=-\frac{m l_{0}^{3} B}{\hbar^{2}} \\
\omega=\frac{m l_{0}^{2} \omega_{1}}{\hbar}
\end{gathered}
$$

and $x$ is measured in units of $l_{0}=1 \mu \mathrm{m}$, a characteristic length unit in this type of experiment, $t$ in units of $m l_{0}^{2} / \hbar$, and $\varphi$ in units of the square root of $n_{0} a^{3}$, the maximum density in the initial distribution of the condensate and the interaction constant is defined as $\mu=-4 \pi n_{0} l_{0}^{2} a$, which is taken positive to the attractive potential function $(a<0)$.

Equation (3) is NLS equation with time-dependent linear potential function. The single dark soliton and single bright soliton [30] are obtained by applying the classical inverse scattering method [31]. The soliton and periodic solutions are also obtained by applying the transformation from the corresponding solution of the NLS equation [32].

In this paper, we apply the similarity transformation [33] and direct hypothesis $[16,34]$ to obtain the analytical rational-like solution of (3) which can describe the possible formation mechanisms of optical rogue waves. In order to make the problem more general, we consider the general form of (3):

$$
i \frac{\partial \varphi}{\partial t}+\frac{1}{2} \frac{\partial^{2} \varphi}{\partial x^{2}}+x f(t) \varphi+\mu|\varphi|^{2} \varphi=0
$$

Without loss of generality, we set $\mu=1$ for $a>0$ and $\mu=-1$ for $a<0$.

\section{Explicit Solutions through Similarity Transformation}

Firstly, we construct the following transformation for the envelope field $\varphi(x, t)$ in the gauge form [33]:

$$
\varphi(x, t)=\left[\varphi_{R}(x, t)+i \varphi_{I}(x, t)\right] e^{i \phi(x, t)},
$$

whose intensity can be written as

$$
|\varphi(x, t)|^{2}=\left|\varphi_{R}(x, t)\right|^{2}+\left|\varphi_{I}(x, t)\right|^{2},
$$

where $\varphi_{R}(x, t), \varphi_{I}(x, t)$ and phase $\phi(x, t)$ are real functions with respect to space $x$ at time $t$.

Substituting (6) into (5) yields the following coupled partial differential equations with variable coefficients:

$$
\begin{aligned}
& -\varphi_{I, t}-\varphi_{R} \phi_{t}+\frac{1}{2} \varphi_{R, x x}-\varphi_{I, x} \phi_{x}-\frac{1}{2} \varphi_{R} \phi_{x}^{2}-\frac{1}{2} \varphi_{I} \phi_{x x} \\
& +\mu\left(\varphi_{R}^{2}+\varphi_{I}^{2}\right) \varphi_{R}+x f \varphi_{R}=0, \\
& \varphi_{R, t}-\varphi_{I} \phi_{t}+\frac{1}{2} \varphi_{I, x x}+\varphi_{R, x} \phi_{x}-\frac{1}{2} \varphi_{I} \phi_{x}^{2}+\frac{1}{2} \varphi_{R} \phi_{x x} \\
& +\mu\left(\varphi_{R}^{2}+\varphi_{I}^{2}\right) \varphi_{I}+x f \varphi_{I}=0 .
\end{aligned}
$$

For the real functions $\varphi_{R}(x, t), \varphi_{I}(x, t)$, and $\phi(x, t)$, introducing the new variables $\eta(x, t)$ and $\tau(t)$ and further utilizing the similarity transformations, we have

$$
\begin{aligned}
\varphi_{R} & =A(t)+B(t) P(\eta(x, t), \tau(t)), \\
\varphi_{I} & =C(t) Q(\eta(x, t), \tau(t)), \\
\phi & =\chi(x, t)+\lambda \tau(t)
\end{aligned}
$$

where $\lambda$ is a constant.

Substituting (9a), (9b), and (9c) into (8a) and (8b), we obtain the following equations:

$$
\begin{aligned}
- & C_{t} Q-C\left(Q_{\eta} \eta_{t}+Q_{\tau} \tau_{t}\right)-(A+B P)\left(\chi_{t}+\lambda \tau_{t}\right) \\
& +\frac{B}{2}\left(P_{\eta \eta} \eta_{x}^{2}+P_{\eta} \eta_{x x}\right)-C Q_{\eta} \eta_{x} \chi_{x}-\frac{1}{2}(A+B P) \chi_{x}^{2} \\
& -\frac{1}{2} C Q \chi_{x x}+\mu\left[(A+B P)^{2}+C^{2} Q^{2}\right](A+B P) \\
& +x f(A+B P)=0,
\end{aligned}
$$




$$
\begin{aligned}
A_{t} & +B_{t} P-B\left(P_{\eta} \eta_{t}+P_{\tau} \tau_{t}\right)-C Q\left(\chi_{t}+\lambda \tau_{t}\right) \\
& +\frac{C}{2}\left(P_{\eta \eta} \eta_{x}^{2}+P_{\eta} \eta_{x x}\right)-B P_{\eta} \eta_{x} \chi_{x}-\frac{1}{2} C Q \chi_{x}^{2} \\
& -\frac{1}{2}(A+B P) \chi_{x x}+\mu\left[(A+B P)^{2}+C^{2} Q^{2}\right] C Q \\
& +x f C Q=0 .
\end{aligned}
$$

Simplifying the above equations, we obtain the similarity reduction

$$
\begin{aligned}
& \eta_{x x}=0 \\
& \eta_{t}+\eta_{x} \chi_{x}=0, \\
& \sigma_{t}+\frac{\sigma}{2} \chi_{x x}=0, \quad(\sigma=A, B, C) \\
& \chi_{t}+\frac{1}{2} \chi_{x}^{2}-x f=0 \\
& -C Q_{\tau} \tau_{t}-(A+B P) \lambda \tau_{t}+\frac{B}{2} P_{\eta \eta} \eta_{x}^{2} \\
& \quad+\mu(A+B P)\left[(A+B P)^{2}+C^{2} Q^{2}\right]=0 \\
& -B P_{\tau} \tau_{t}-C Q \lambda \tau_{t}+\frac{C}{2} Q_{\eta \eta} \eta_{x}^{2} \\
& \quad+\mu C Q\left[(A+B P)^{2}+C^{2} Q^{2}\right]=0
\end{aligned}
$$

where $\eta(x, t), \chi(x, t), A(t), B(t), C(t), P(\eta, \tau)$, and $Q(\eta, \tau)$ are the functions to be determined.

After some algebra computation, it follows from (11a)(11d) that we have

$$
\begin{aligned}
\eta(x, t) & =\alpha(t) x+\beta(t), \\
\chi(x, t) & =-\frac{\alpha_{t}}{2 \alpha} x^{2}-\frac{\beta_{t}}{\alpha} x+\chi_{0}(t), \\
A(t) & =a_{0} \sqrt{|\alpha|}, \\
B(t) & =b A, \\
C(t) & =d A
\end{aligned}
$$

where $a_{0}, b$, and $d$ are constants, $\alpha(t)$ is the inverse of the wave width, and $-\beta(t) / \alpha(t)$ is the position of its center of mass. $\alpha(t), \beta(t)$, and $\chi_{0}(t)$ are all free functions with respect to time t.

To further reduce (11e)-(11f) to the coupled partial differential equations, we require

$$
\tau(t)=\frac{1}{2} \int_{0}^{t} \alpha^{2}(s) \mathrm{ds}, \quad \alpha^{2}=2 A^{2} .
$$

Therefore, (11a), (11b), (11c), (11d), (11e), and (11f) are reduced to

$$
\begin{aligned}
& -d Q_{\tau}-(1+b P) \lambda+b P_{\eta \eta} \\
& \quad+\mu(1+b P)\left[(1+b P)^{2}+d^{2} Q^{2}\right]=0, \\
& -b P_{\tau}-d Q \lambda+d Q_{\eta \eta}+\mu d Q\left[(1+b P)^{2}+d^{2} Q^{2}\right]=0 .
\end{aligned}
$$

For simplicity, we set $\mu=1$. According to the direct method developed in $[14,15]$, we obtain the first-order rational solution of (14a) and (14b)

$$
\begin{gathered}
P(\eta, \tau)=-\frac{4}{R_{1}(\eta, \tau) b}, \\
Q(\eta, \tau)=-\frac{8 \tau}{R_{1}(\eta, \tau) d},
\end{gathered}
$$

where $R_{1}(\eta, \tau)=1+2 \eta^{2}+4 \tau^{2}$ and the second-order rational solution of (14a) and (14b) is given

$$
\begin{gathered}
P(\eta, \tau)=\frac{P_{1}(\eta, \tau)}{R_{2}(\eta, \tau) b}, \\
Q(\eta, \tau)=\frac{Q_{1}(\eta, \tau) \tau}{R_{2}(\eta, \tau) d},
\end{gathered}
$$

where

$$
\begin{aligned}
P_{1}(\eta, \tau)= & \frac{3}{8}-9 \tau^{2}-\frac{3}{2} \eta^{2}-6 \eta^{2} \tau^{2}-10 \tau^{4}-\frac{1}{2} \eta^{4} \\
Q_{1}(\eta, \tau)= & -\frac{15}{4}+2 \tau^{2}-3 \eta^{2}+4 \eta^{2} \tau^{2}+4 \tau^{4}+\eta^{4} \\
R_{2}(\eta, \tau)= & \frac{3}{32}+\frac{33}{8} \tau^{2}+\frac{9}{16} \eta^{2}-\frac{3}{2} \eta^{2} \tau^{2}+\frac{9}{2} \tau^{4} \\
& +\frac{1}{8} \eta^{4}+\frac{2}{3} \tau^{6}+\eta^{2} \tau^{6}+\frac{1}{2} \eta^{4} \tau^{2} \\
& +\frac{1}{12} \eta^{6}
\end{aligned}
$$

Thus, a direct reduction solution of (5) can be derived as

$$
\varphi=A(1+b P+i d Q) e^{i(\chi+\tau)},
$$

where $A(t), P(\eta, \tau), Q(\eta, \tau), \chi(x, t)$, and $\tau(t)$ are expressed by (12a), (12b), (12c), (13), and (16), respectively.

\section{Rogue Waves Solutions}

3.1. First-Order Solution. Now we focus on the rogue wave structures in the solutions of the nonlinear Schrödinger equation with time-dependent linear potential function. Substituting (15) into (18), we have the first-order rational-like solution of (5):

$$
\varphi=a_{0} \sqrt{|\alpha|}\left(1-\frac{4+8 i \tau}{1+2 \eta^{2}+4 \tau^{2}}\right) e^{i(\chi+\tau)},
$$




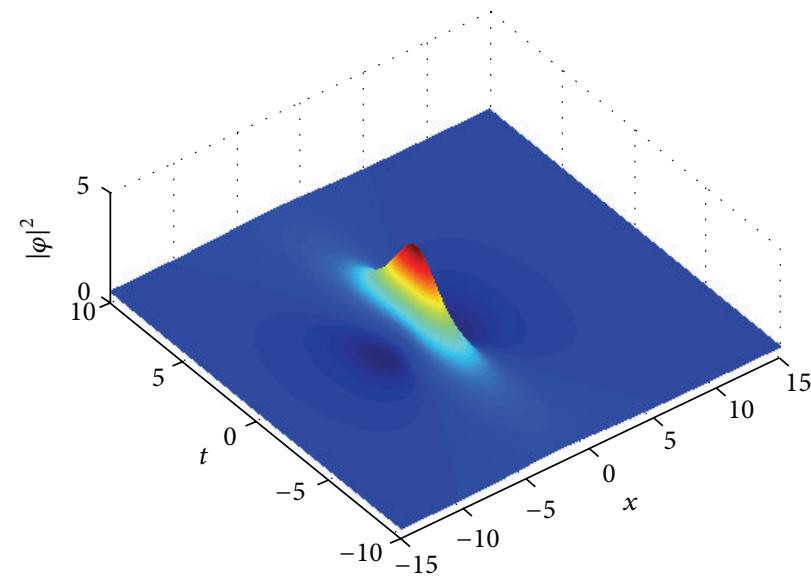

Figure 1: The first-order rogue wave propagations for the intensity $|\varphi|^{2}$ with the gravity field $b=0.5$.

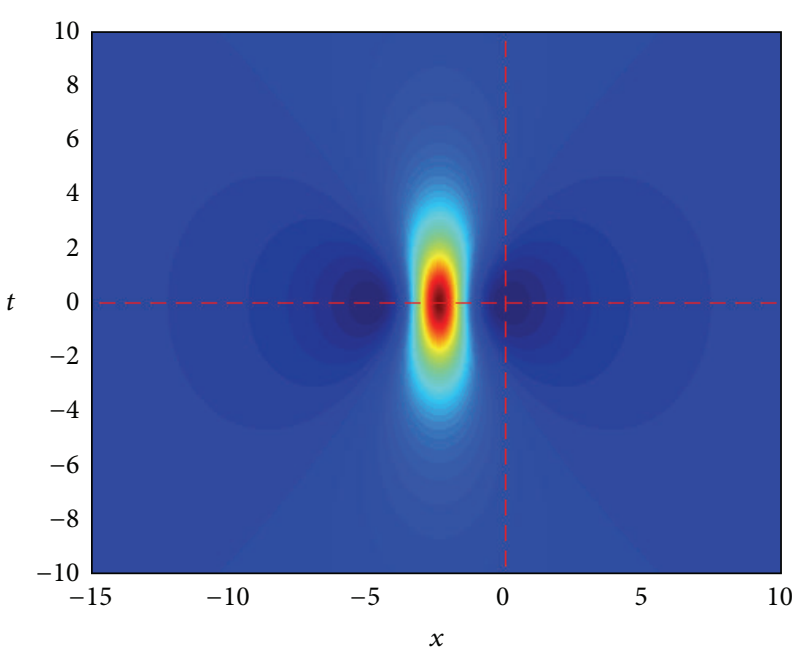

(a)

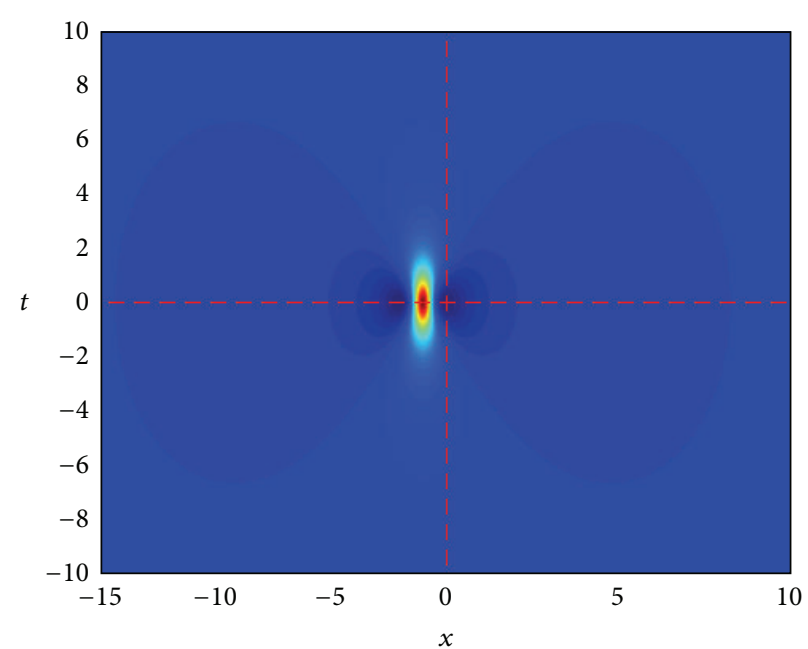

(b)

FIGURE 2: Contour plots of the first-order rogue wave propagation with the gravity fields (a) $b=0.5$; (b) $b=1.2$.

whose amplitude can be written as

$$
\begin{aligned}
|\varphi|^{2} & =a_{0}^{2}|\alpha(t)| \\
& \frac{\left[2(\alpha(t) x+\beta(t))^{2}+4 \tau^{2}-3\right]^{2}+64 \tau^{2}(t)}{\left[1+2(\alpha(t) x+\beta(t))^{2}+4 \tau^{2}(t)\right]^{2}}
\end{aligned}
$$

It can be seen that the structure of solution (19) is different from one of the solutions obtained in $[29,31]$. In what follows, we will choose the function $\alpha(t)=f(t)=b+l \cos (\omega t)$ to exhibit the nonlinear dynamic behaviors of the rogue waves which change with the gravity field $b=\delta m g$ and timedependent external magnetic field $l \cos (\omega t)$, where $\delta$ is a constant.
We study the nonlinear dynamic behaviors of the rogue waves when there is only the gravity field; namely, $l=0$. Thus, the amplitude given by (20) is changed to

$$
|\varphi|^{2}=a_{0}^{2} b^{2} \frac{\left[2(b x+\beta(t))^{2}+4 \tau^{2}-3\right]^{2}+64 \tau^{2}(t)}{\left[1+2(b x+\beta(t))^{2}+4 \tau^{2}(t)\right]^{2}}
$$

where $\tau(t)=(1 / 2) b t$.

We plot figures of the amplitude using (21) with different value $b$ and function $\beta(t)$.

Case 1. Supposing that free function $\beta(t)$ is a constant and setting the gravity field $b=0.5$, the structure of the rogue wave described by (21) is plotted, as shown in Figure 1. For better understanding the structure of the rogue wave, $b=$ 0.5 and 1.2. The contour plots of the first-order rogue wave propagation are depicted, as shown in Figures 2(a)-2(b), 


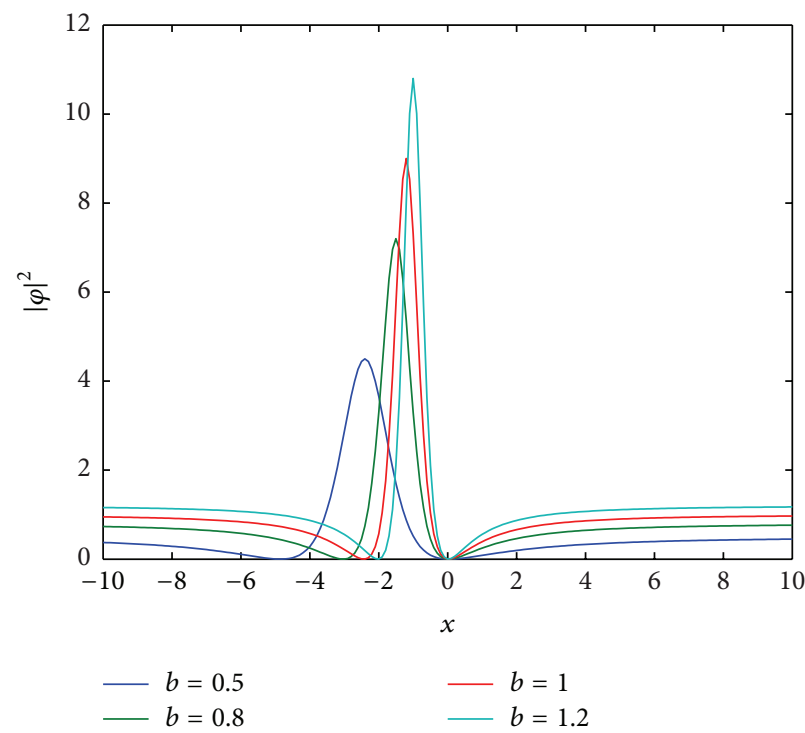

(a)

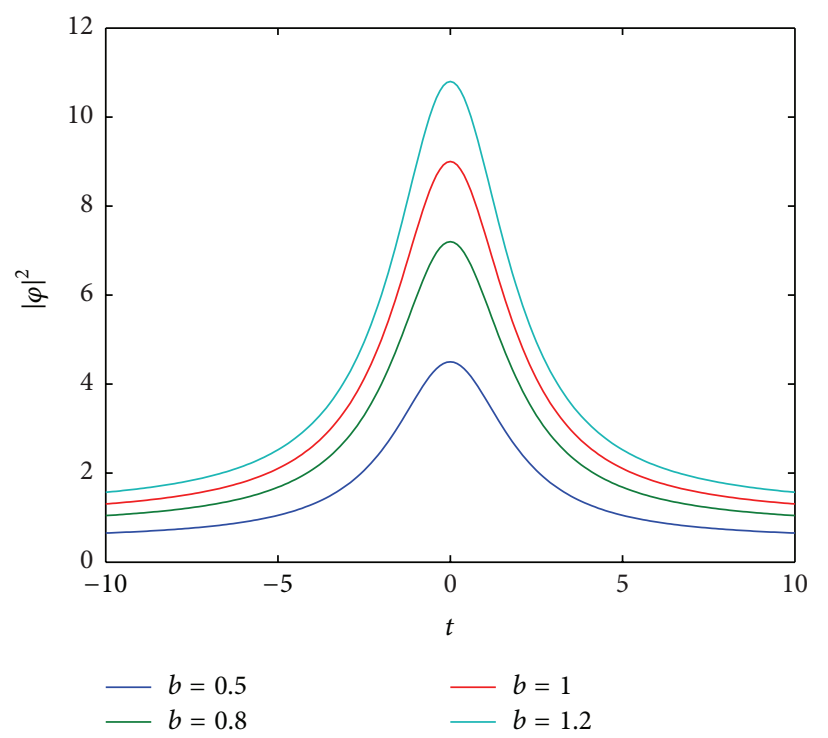

(b)

Figure 3: (a) Coordinate variation of the amplitude of the first-order rogue waves with the gravity fields $b=0.5, b=0.8, b=1.2$, and $b=1.5$. (b) Time variation of the amplitude of the first-order rogue waves with the gravity fields $b=0.5, b=0.8, b=1.2$, and $b=1.5$.

respectively. It can be observed from Figures 2(a)-2(b) that the pattern of the rogue waves is the same when the gravity field $b$ takes any value. However, it is found from Figures 2(a)2(b) that the position $x$, at which the highest amplitudes of the rogue waves occur, is different. Suppose $b=0.8$ and $b=1.5$; the relations on the rogue waves amplitude via coordinate and time are, respectively, obtained, as shown in Figure 3. It is clearly seen from Figure 3(a) that, with increasing of the gravity field, the amplitude of the rogue waves has an increase in the peak density. The maximum amplitude of the rogue waves occurs at the points $x=-2.4,-1.5,-1.2$, and -1 . It is also observed from Figure $3(\mathrm{~b})$ that the time, on which the maximum amplitude of the rogue waves occurs, is the same, where $t=0$.

Case 2. Suppose that the free function $\beta(t)$ is the polynomial with respect to time $t$, namely, $\beta(t)=0.2 t^{2}$, and the compound function $\beta(t)=0.5 \exp \left(\operatorname{sech}\left(0.2 t^{3}\right)\right)$. Then, Figures $4(\mathrm{a})$ and $4(\mathrm{c})$ give the nonlinear dynamical behaviors of the rational-like solution (21). When $t=0$, the changing cases of $|\varphi(x, 0)|^{2}$ via $x$ are depicted, as shown in Figures $4(\mathrm{~b})$ and 4(d). In Figure 4(b), the position of maximum amplitude $|\varphi|^{2}$ appears at $x=0$. However, in Figure 4(d), the position of maximum amplitude $|\varphi|^{2}$ appears at $x=-2.7$. In two cases, it is found from Figures $4(\mathrm{~b})$ and $4(\mathrm{~d})$ that the maximum amplitudes equal 4.5. If different free functions $\beta(t)$ are chosen, the solution determined by (21) is of different dynamic properties.

In the following analysis, we study the nonlinear dynamic behaviors of the rogue waves when there exist the gravity field and external magnetic field; namely, $l \neq 0$. For simplicity, we suppose $b=0.8+1.2 \cos (0.005 t)$ and $\beta=0.2 t^{2}$. The nonlinear dynamical behavior of the rational-like solution (21) is plotted, as shown in Figure 5(a). When $t=0$, the changing case of $|\varphi(x, 0)|^{2}$ via $x$ is depicted, as shown in Figure 5(b). In Figure 5(b), the position of maximum amplitude $|\varphi|^{2}$ appears at $x=0$ and the maximum amplitude is equal to 18 . Comparing the amplitudes of Figure 5(b) with Figure 4(b), we observe that the amplitude of the rogue wave has a large and quick increase when the external magnetic field exists, as shown in Figure 5(c). The amplitudes $|\varphi(x, 0)|^{2}$ reach the largest at the point $x=0$. Furthermore, we adjust the intensity $l$ of the external magnetic field to analyze the changing cases of the amplitude for the rogue waves.

3.2. Second-Order Solution. Substituting (16) into (18), we obtain the second-order rational-like solution of (5)

$$
\varphi=a_{0} \sqrt{|\alpha|}\left(1+\frac{P_{1}}{R_{2}}+i \frac{Q_{1} \tau}{R_{2}}\right) e^{i(\chi+\tau)},
$$

whose intensity is written as

$$
|\varphi|^{2}=a_{0}^{2}|\alpha(t)|\left[\left(1+\frac{P_{1}}{R_{2}}\right)^{2}+\left(\frac{Q_{1} \tau}{R_{2}}\right)^{2}\right],
$$

where $P_{1}(\eta, \tau), Q_{1}(\eta, \tau)$, and $R_{2}(\eta, \tau)$ are expressed by (17a)(17c), respectively.

The effect of the gravity field on the rogue wave is studied. Similar to the first-order solution, we suppose $b=0.5$ and $\beta(t)=0.2$. The nonlinear dynamic behaviors of the secondorder rogue wave are depicted, as shown in Figures 6(a)-6(b). Compared with the first-order solution, it is found that there 


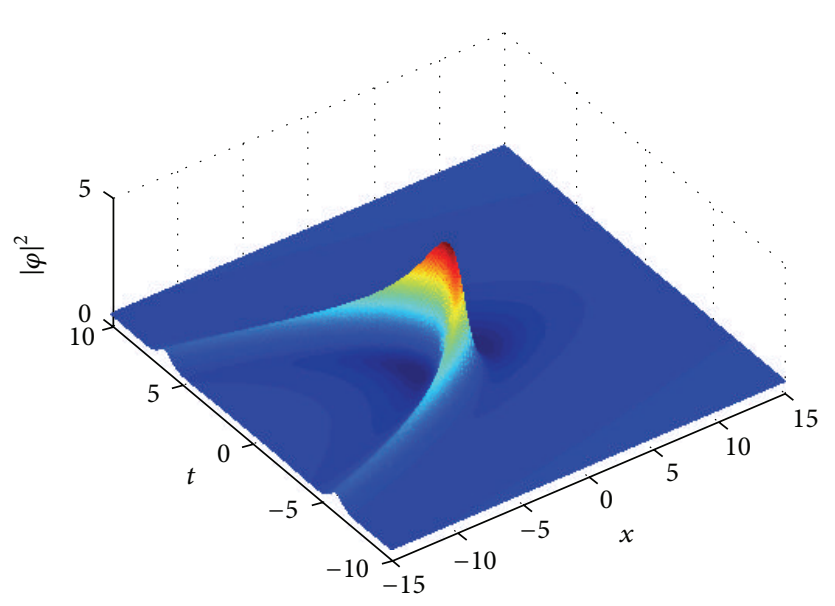

(a)

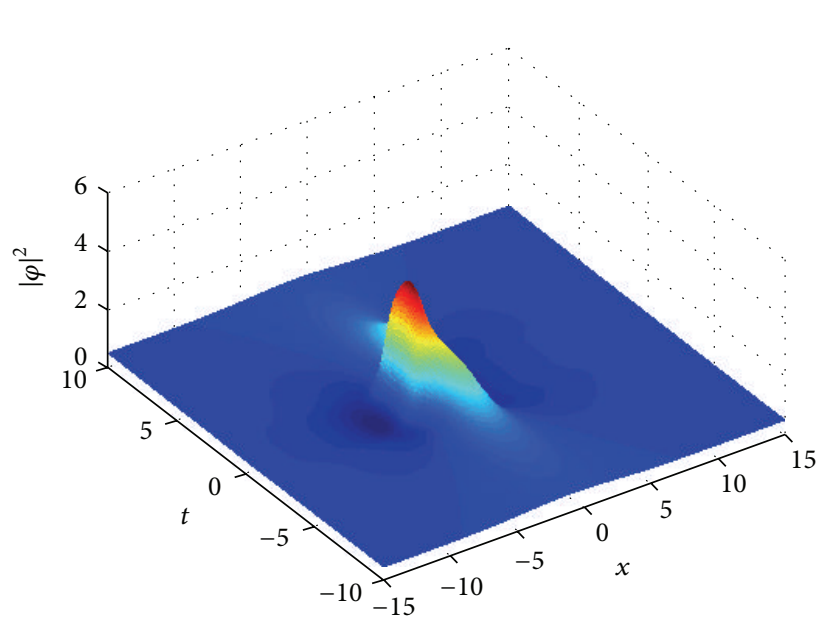

(c)

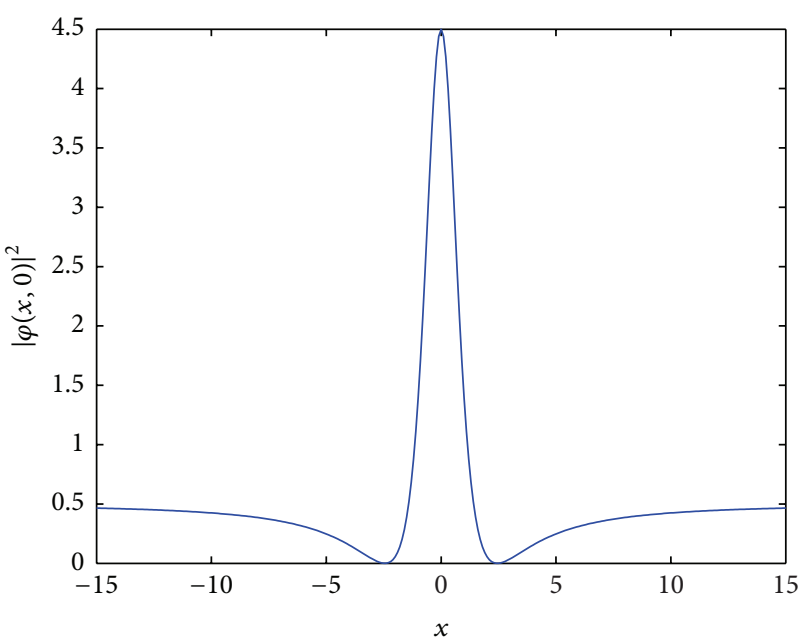

(b)

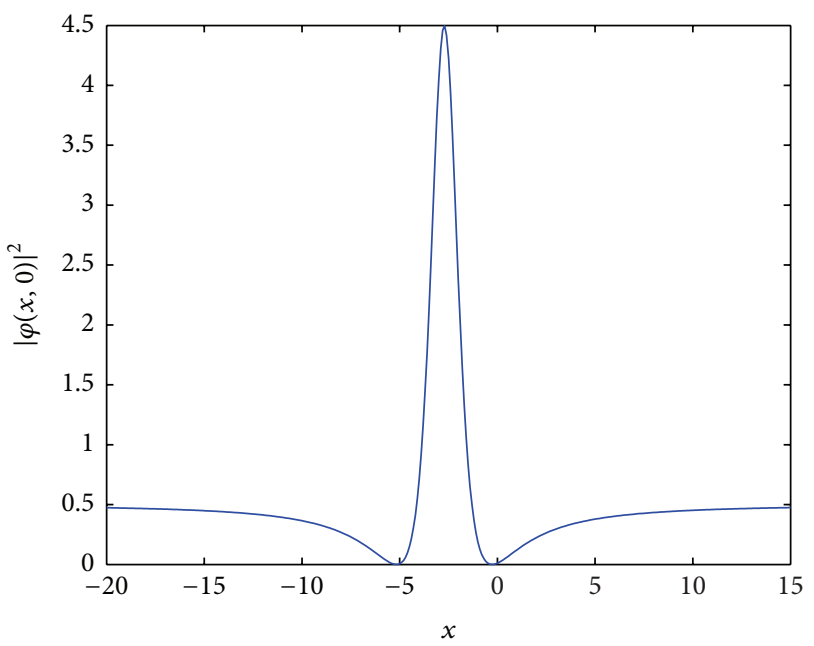

(d)

FIGURE 4: The first-order rogue waves propagation for the intensity $|\varphi|^{2}$ with the gravity field $b=0.5$ and (a)-(b) $\beta(t)=0.2 t^{2},(\mathrm{c})-(\mathrm{d})$ $\beta(t)=0.5 \exp \left(\operatorname{sech}\left(0.2 t^{3}\right)\right)$.

are four small peaks around one high peak in the secondorder rogue waves and all energy of the rogue wave is focused on the high peak. The amplitude of the second-order rationallike solution is even larger than the one of the first-order solution. It is observed from Figure 6(a) that the maximum amplitude equals 12.5 .

Suppose $b=0.5$ and $\beta(t)=0.5 \exp \left(\operatorname{sech}\left(0.2 t^{3}\right)\right)$; the nonlinear dynamic behaviors of the second-order rogue waves are also depicted, as shown in Figures 7(a)-7(b). When $t=0$, the changing procedure of $|\varphi(x, 0)|^{2}$ via $x$ is depicted, as shown in Figure 7(c). In Figure 7(c), the position of maximum amplitude $|\varphi|^{2}$ appears at $x=-2.7$. It is observed that the maximum amplitude equals 12.48.

Then, we also study the nonlinear dynamic behaviors of the second-order rogue waves when there exist the gravity field and external magnetic field. Suppose $\alpha(t)=0.5+$ $1.2 \cos (8 t)$ and $\beta(t)=0.2 t$; the nonlinear dynamic behaviors of the second-order rogue waves are depicted, as shown in Figure 8. It is observed from Figure 8 that the second-order rogue wave is different from the ones displayed in Figures 6(a) and $7(\mathrm{a})$. In this case, the wave width is thin and the rogue wave changes more quickly than the first-order one displayed in Figure 5(a). The maximum amplitude equals 45.

\section{Conclusion}

In this paper, we present the rogue waves of the nonlinear Schrödinger equation with time-dependent linear potential function. Using the similarity transformation and direct hypothesis, we obtain the first-order and second-order solutions of the rogue waves and discuss the nonlinear dynamic behaviors of these solutions in detail. In addition, we also analyze effect of the gravity field and external magnetic field changing with the time on the amplitudes of the rogue waves. It is found that the gravity field and external magnetic field have significant effect on the amplitude of the rogue waves. The numerical results obtained in this paper are useful to study the matter rogue waves in the Bose-Einstein condensates and other fields of nonlinear science. 


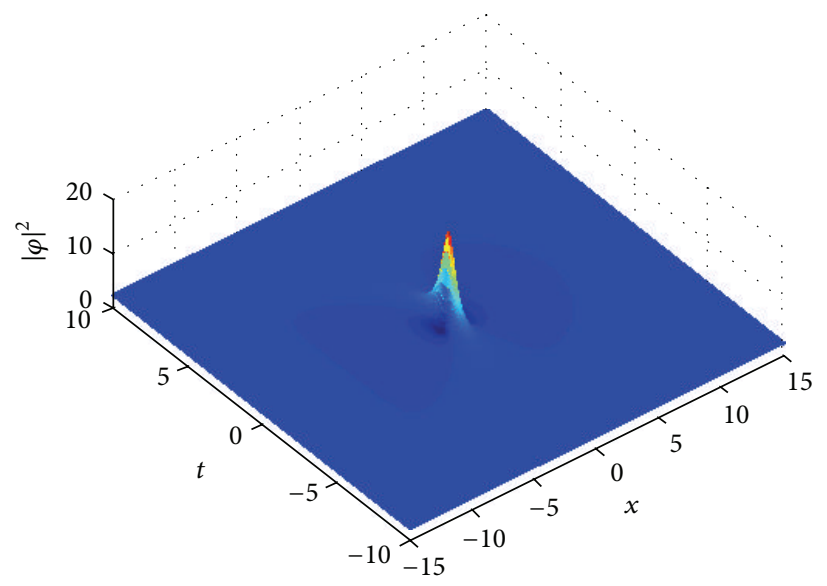

(a)

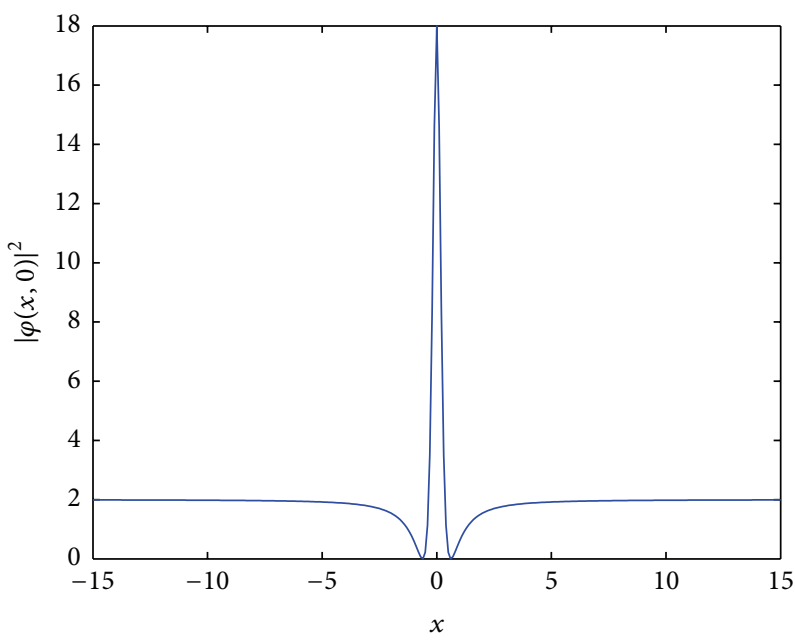

(b)

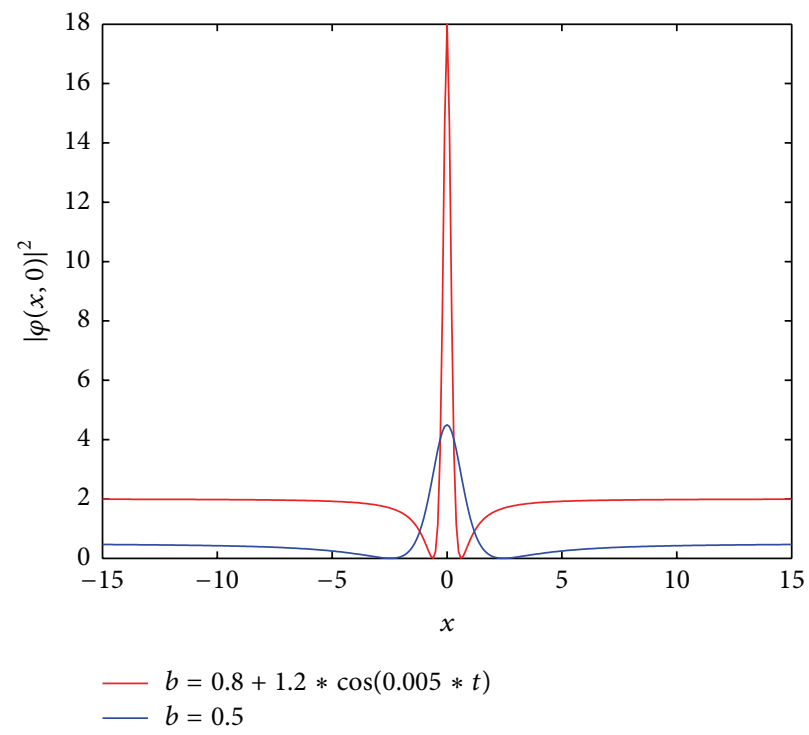

(c)

Figure 5: (a) The first-order rogue wave propagations for the intensity $|\varphi|^{2}$ with the gravity field and external magnetic field $b=0.8+$ $1.2 \cos (0.005 t)$ and $\beta=0.2 t^{2}$. (b) Rogue wave propagations for the intensity $|\varphi(x, 0)|^{2}$ with $t=0$. (c) The comparison of the amplitude between $b=0.8$ and $b=0.8+1.2 \cos (0.005 t)$.

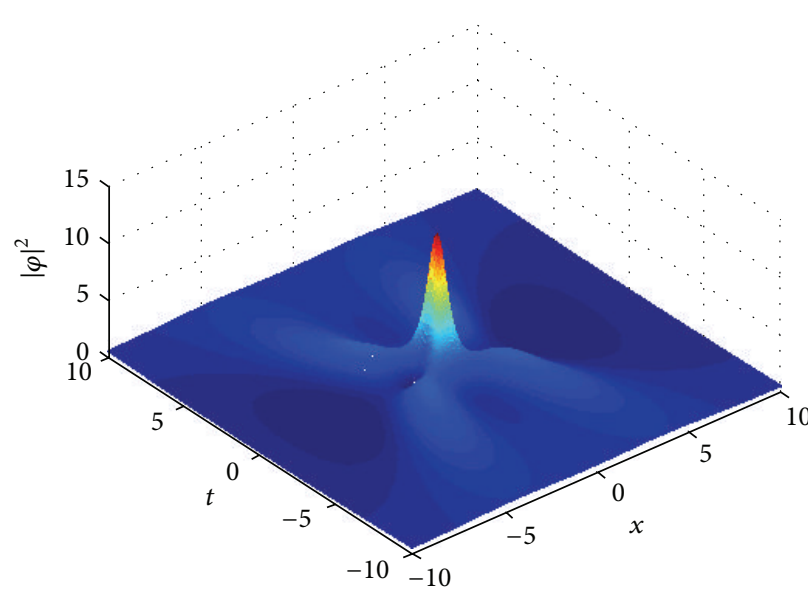

(a)

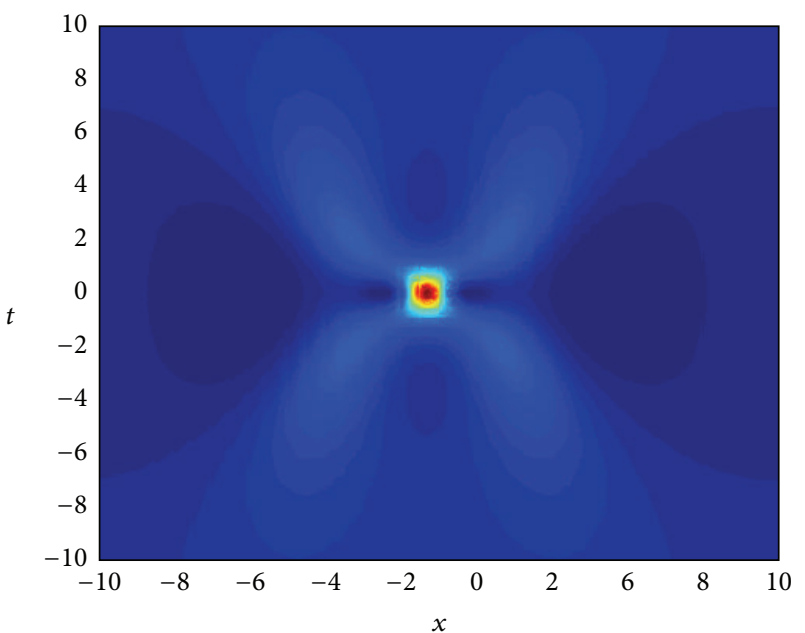

(b)

Figure 6: (a) The second-order rogue wave propagations for the intensity $|\varphi|^{2}$. (b) Contour plot with the gravity field $b=0.5, \beta(t)=0.2$. 


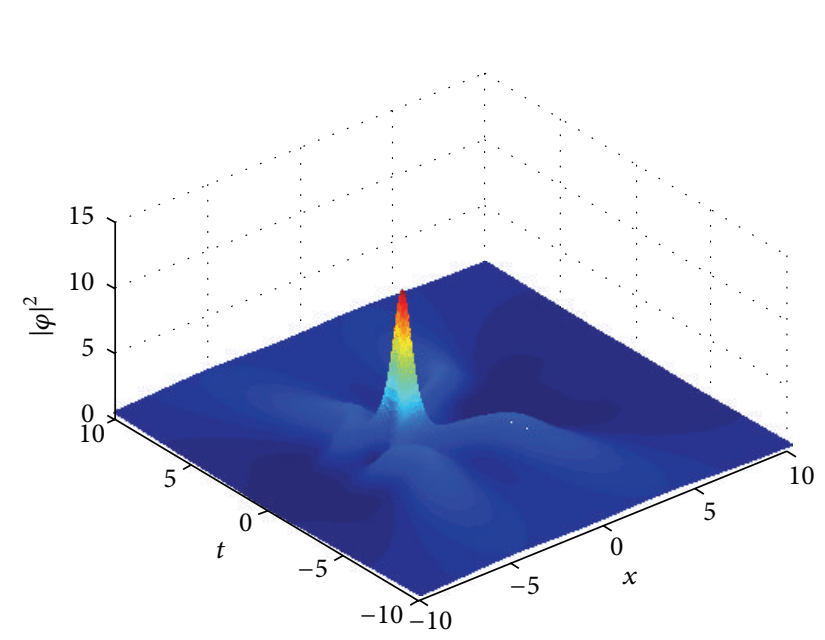

(a)

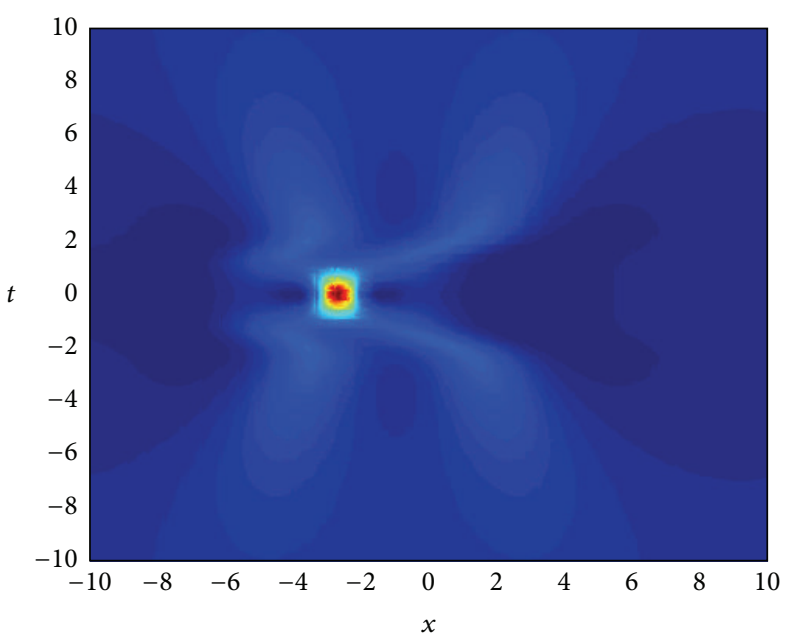

(b)

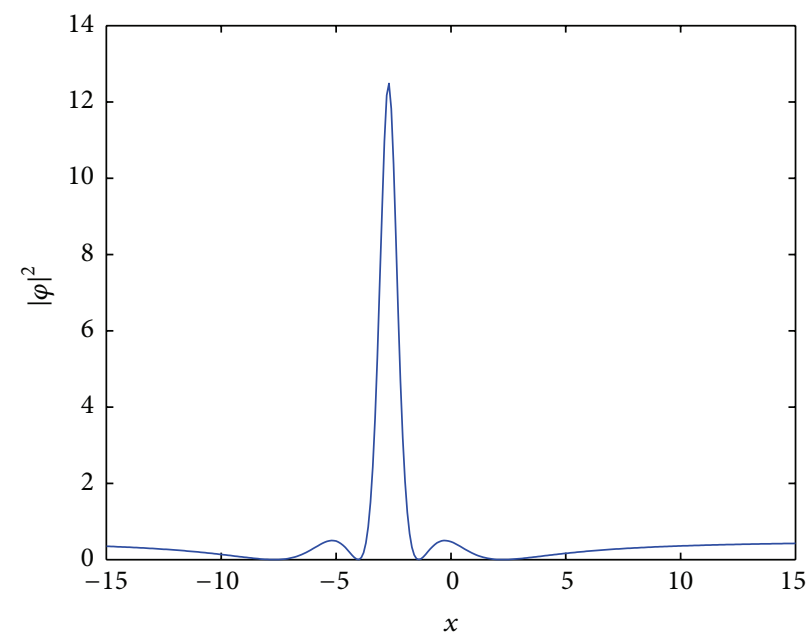

(c)

Figure 7: (a) The second-order rogue wave propagations for the intensity $|\varphi|^{2}$. (b) Contour plot with the gravity field $b=0.5, \beta(t)=$ $0.5 \exp \left(\operatorname{sech}\left(0.2 t^{3}\right)\right)$. (c) Rogue wave propagations for the intensity $|\varphi(x, 0)|^{2}$ with $t=0$.

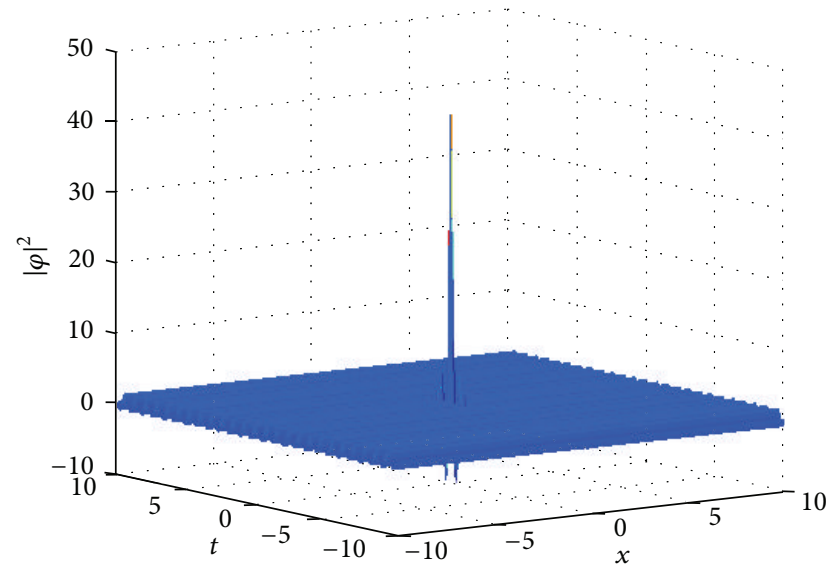

FIGURE 8: The second-order rogue wave propagations for the intensity $|\varphi|^{2}$ with the gravity field and external magnetic field $\alpha(t)=$ $0.5+1.2 \cos (8 t)$.

\section{Conflict of Interests}

The authors declare that there is no conflict of interests regarding the publication of this paper.

\section{Acknowledgments}

The authors thank sincerely Professor Wei Zhang for many useful discussions. This work is supported by the National Sciences Foundation of Shanxi Province (2015011009).

\section{References}

[1] P. Müller, C. Garrett, and A. Osborne, "Rogue waves-the fourteenth 'Aha Huliko'a Hawaiian Winter Workshop," Oceanography, vol. 18, no. 3, pp. 66-75, 2005.

[2] N. Akhmediev, A. Ankiewicz, and M. Taki, "Waves that appear from nowhere and disappear without a trace," Physics Letters A, vol. 373, no. 6, pp. 675-678, 2009. 
[3] D. R. Solli, C. Ropers, P. Koonath, and B. Jalali, "Optical rogue waves," Nature, vol. 450, no. 7172, pp. 1054-1057, 2007.

[4] Y. V. Bludov, V. V. Konotop, and N. Akhmediev, "Matter rogue waves," Physical Review A, vol. 80, no. 3, Article ID 033610, 2009.

[5] A. N. Ganshin, V. B. Efimov, G. V. Kolmakov, L. P. MezhovDeglin, and P. V. E. McClintock, "Observation of an inverse energy cascade in developed acoustic turbulence in superfluid helium," Physical Review Letters, vol. 101, no. 6, Article ID 065303, 2008.

[6] C. Kharif and E. Pelinovsky, "Physical mechanisms of the rogue wave phenomenon," European Journal of Mechanics. B. Fluids, vol. 22, no. 6, pp. 603-634, 2003.

[7] P. A. E. M. Janssen, "Nonlinear four-wave interactions and freak waves," Journal of Physical Oceanography, vol. 33, no. 4, pp. 863884, 2003.

[8] M. Onorato, A. R. Osborne, M. Serio, and S. Bertone, "Freak waves in random oceanic sea states," Physical Review Letters, vol. 86, no. 25, pp. 5831-5834, 2001.

[9] C.-Q. Dai, Y.-Y. Wang, Q. Tian, and J.-F. Zhang, "The management and containment of self-similar rogue waves in the inhomogeneous nonlinear Schrödinger equation," Annals of Physics, vol. 327, no. 2, pp. 512-521, 2012.

[10] X.-L. Wang, W.-G. Zhang, B.-G. Zhai, and H.-Q. Zhang, "Rogue waves of the higher-order dispersive nonlinear Schrödinger equation," Communications in Theoretical Physics, vol. 58, no. 4, pp. 531-538, 2012.

[11] Y. Y. Wang and C. Q. Dai, "Spatiotemporal Rogue waves for the Variable-coefficient $(3+1)$-dimensional nonlinear Schrödinger equation," Communications in Theoretical Physics, vol. 58, no. 2, pp. 255-260, 2012.

[12] X.-C. Wang, J.-S. He, and Y.-S. Li, "Rogue wave with a controllable center of nonlinear Schrödinger equation," Communications in Theoretical Physics, vol. 56, no. 4, pp. 631-637, 2011.

[13] Z.-Y. Yan, "Financial rogue waves," Communications in Theoretical Physics, vol. 54, no. 5, pp. 947-949, 2010.

[14] D. H. Peregrine, "Water waves, nonlinear Schrödinger equations and their solutions," Journal of the Australian Mathematical Society, Series B, vol. 25, no. 1, pp. 16-43, 1983.

[15] N. Akhmediev, A. Ankiewicz, and J. M. Soto-Crespo, "Rogue waves and rational solutions of the nonlinear Schrödinger equation," Physical Review E, vol. 80, no. 2, Article ID 026601, 2009.

[16] N. Akhmediev, J. M. Soto-Crespo, and A. Ankiewicz, "Extreme waves that appear from nowhere: on the nature of rogue waves," Physics Letters A, vol. 373, no. 25, pp. 2137-2145, 2009.

[17] Z. Y. Yan, "Nonautonomous 'rogons' in the inhomogeneous nonlinear Schrödinger equation with variable coefficients," Physics Letters A, vol. 374, no. 4, pp. 672-679, 2010.

[18] S. Y. Song, J. Wang, J. M. Meng, J. B. Wang, and P. X. Hu, "Nonlinear Schrödinger equation for internal waves in deep sea," Acta Physica Sinica, vol. 59, no. 2, pp. 1123-1129, 2010.

[19] D. H. Peregrine, "Water waves, nonlinear Schrödinger equations and their solutions," The Journal of the Australian Mathematical Society Series B: Applied Mathematics, vol. 25, no. 1, pp. 16-43, 1983.

[20] X. P. Cheng, J. Y. Wang, and J. Y. Li, "Controllable rogue waves in coupled nonlinear Schrödinger equations with varying potentials and nonlinearities," Nonlinear Dynamics, vol. 77, no. 3, pp. 545-552, 2014.

[21] X.-F. Wu, G.-S. Hua, and Z.-Y. Ma, "Evolution of optical solitary waves in a generalized nonlinear Schrödinger equation with variable coefficients," Nonlinear Dynamics, vol. 70, no. 3, pp. 2259-2267, 2012.

[22] M. H. Anderson, J. R. Ensher, M. R. Matthews, C. E. Wieman, and E. A. Cornell, "Observation of Bose-Einstein condensation in a dilute atomic vapor," Science, vol. 269, no. 5221, pp. 198-201, 1995.

[23] C. C. Bradley, C. A. Sackett, J. J. Tollett, and R. G. Hulet, "Evidence of Bose-Einstein condensation in an atomic gas with attractive interactions," Physical Review Letters, vol. 75, no. 9, pp. 1687-1690, 1995.

[24] K. B. Davis, M.-O. Mewes, M. R. Andrews et al., "Bose-Einstein condensation in a gas of sodium atoms," Physical Review Letters, vol. 75, no. 22, pp. 3969-3973, 1995.

[25] V. G. Ivancevic and D. J. Reid, “Turbulence and shock-waves in crowd dynamics," Nonlinear Dynamics, vol. 68, no. 1-2, pp. 285304, 2012.

[26] H.-P. Zhu, "Nonlinear tunneling for controllable rogue waves in two dimensional graded-index waveguides," Nonlinear Dynamics, vol. 72, no. 4, pp. 873-882, 2013.

[27] F. Dalfovo, S. Giorgini, L. P. Pitaevskii, and S. Stringari, “Theory of Bose-Einstein condensation in trapped gases," Reviews of Modern Physics, vol. 71, no. 3, pp. 463-512, 1999.

[28] A. J. Leggett, "Bose-Einstein condensation in the alkali gases: some fundamental concepts," Reviews of Modern Physics, vol. 73, no. 2, pp. 307-356, 2001.

[29] W. B. Fan, Tunneling Transport and Nonlinear Excitations of Bose-Einstein Condensates in the Super Lattice, Institute of Applied Physics and Computational Mathematics, Beijing, China, 2001.

[30] X.-Q. Liu, S. Jiang, W.-B. Fan, and W.-M. Liu, "Soliton solutions in linear magnetic field and time-dependent laser field," Communications in Nonlinear Science and Numerical Simulation, vol. 9, no. 3, pp. 361-365, 2004.

[31] M. J. Ablowitz and P. A. Clarkson, Solitons, Nonlinear Evolution Equations and Inverse Scattering, Cambridge University Press, Cambridge, UK, 1991.

[32] J.-S. He, M. Ji, and Y.-S. Li, "Solutions of two kinds of nonisospectral generalized nonlinear schrödinger equation related to Bose-Einstein condensates," Chinese Physics Letters, vol. 24, no. 8, p. 2157, 2007.

[33] Z. Y. Yan, Constructive Theory and Applications of Complex Nonlinear Waves, Science Press, Beijing, China, 2007.

[34] N. Akhmediev, J. M. Soto-Crespo, and A. Ankiewicz, "How to excite a rogue wave," Physical Review A-Atomic, Molecular, and Optical Physics, vol. 80, no. 4, Article ID 043818, 2009. 


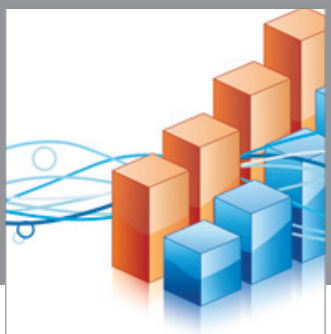

Advances in

Operations Research

vatem alat4

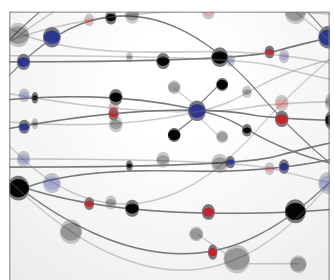

\section{The Scientific} World Journal
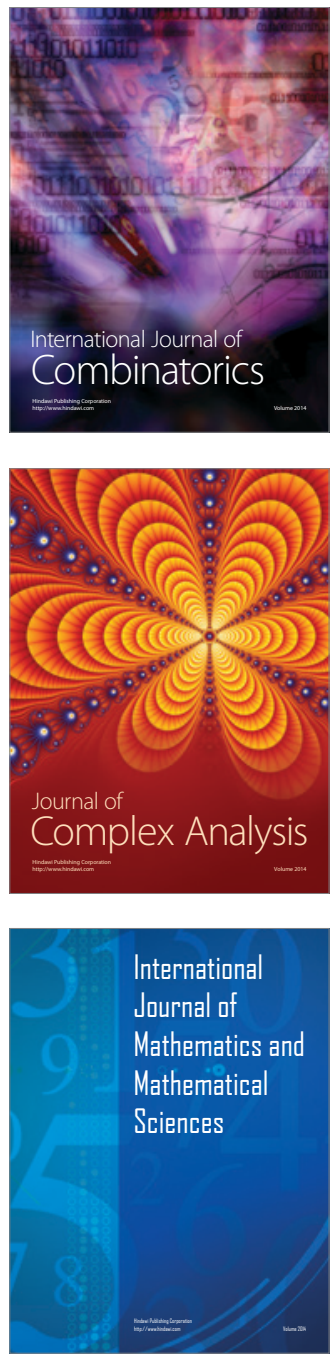
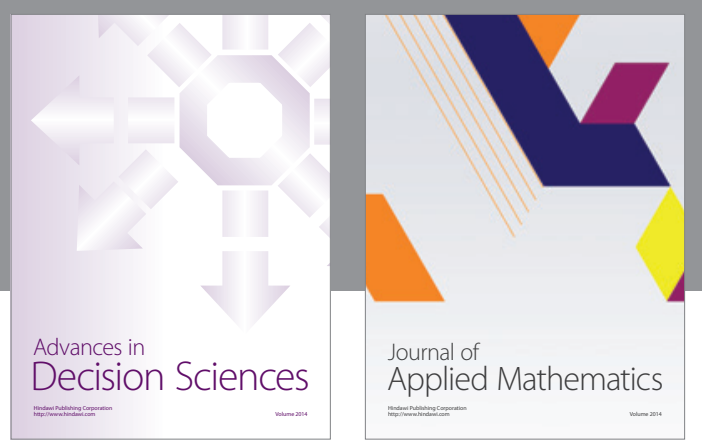

Algebra

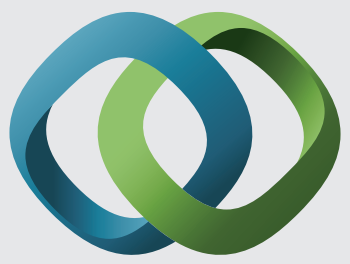

\section{Hindawi}

Submit your manuscripts at

http://www.hindawi.com
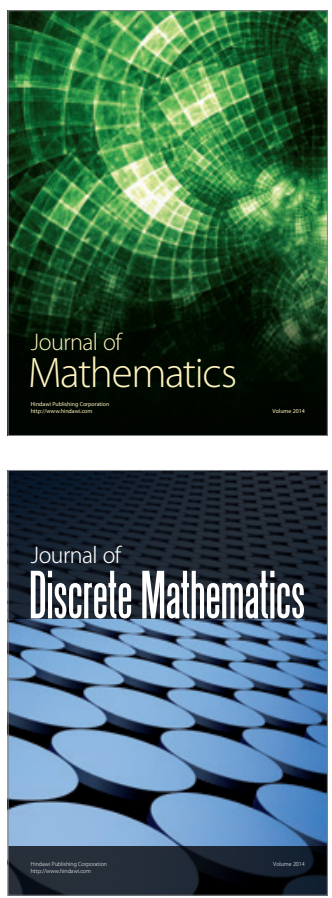

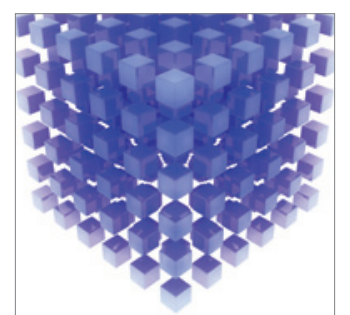

Mathematical Problems in Engineering
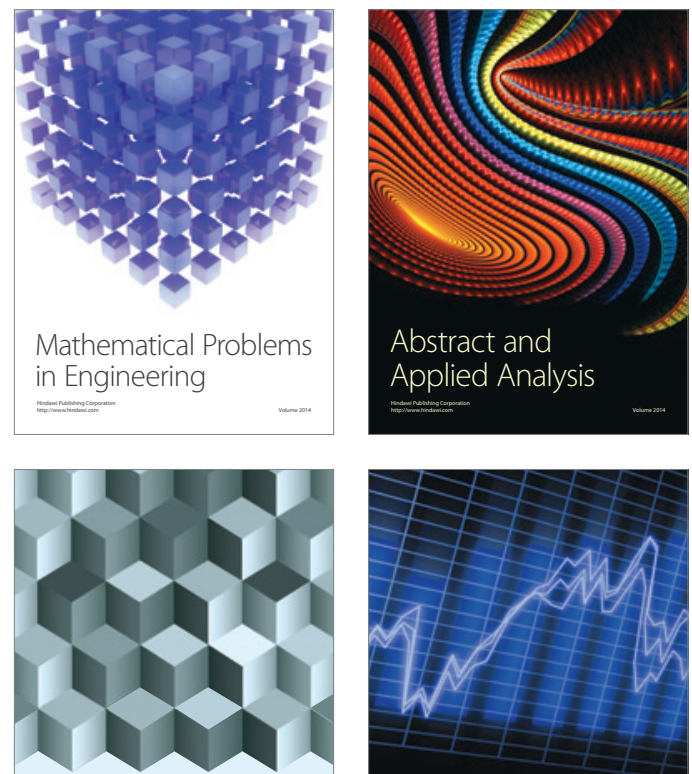

Journal of

Function Spaces

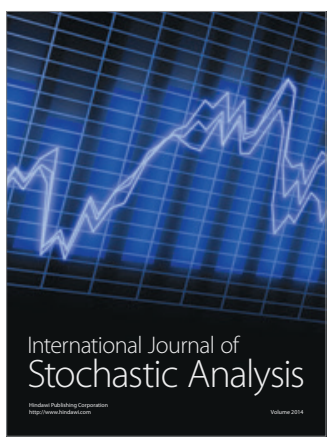

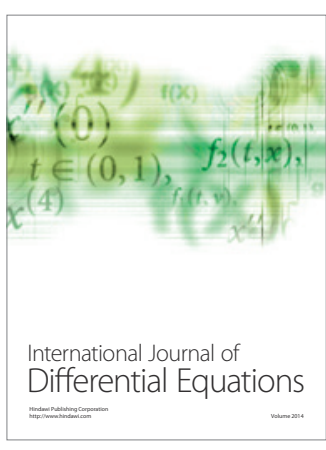
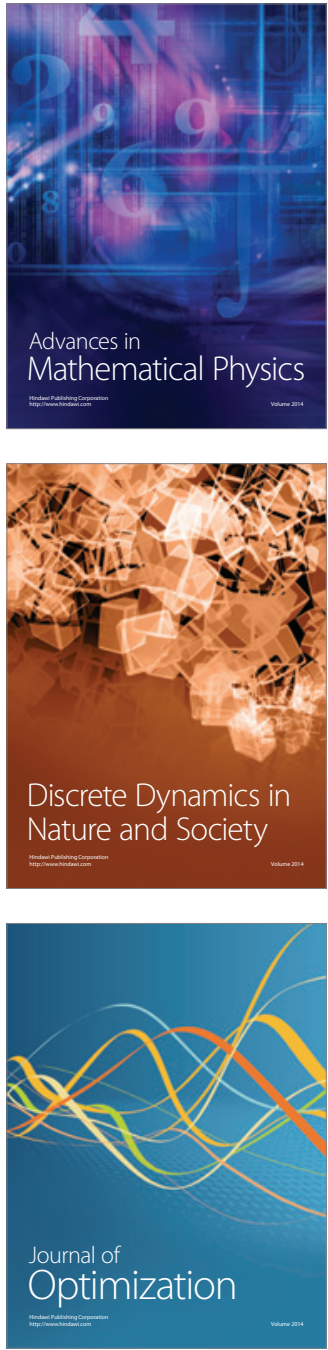